\title{
Learning from Default Mode Network: The Predictive Value of Resting State in Traumatic Brain Injury
}

\author{
Stefano Sandrone ${ }^{1,3}$ and Marco Bacigaluppi ${ }^{2,3}$ \\ ${ }^{1}$ Graduate Program in Psychology-Cognitive Neuroscience, ${ }^{2} \mathrm{PhD}$ Program in Experimental Neurology at Vita-Salute San Raffaele University, and \\ ${ }^{3}$ Neuroimmunology Unit, Institute of Experimental Neurology, Division of Neuroscience, IRCCS San Raffaele Milano, I-20132 Milan, Italy \\ Review of Bonnelle et al.
}

In the 1880s, the Italian physiologist Angelo Mosso hypothesized that an attentional or cognitive task increases cerebral blood flow (Mosso, 1884). Lacking current technology, he tried to measure the blood flow of a supine live subject with a "delicately balanced table which could tip downward either at the head or the foot if the weight of either end were increased" (James, 1890). In resting conditions, the subject was in perfect equilibrium, whereas during emotional or intellectual tasks the head-end of the table inclined downward (Fig. 1). This incredibly simple but revolutionary balance can be considered the first ante litteram "neuroimaging" technique, one that anticipated the physical principles of functional magnetic resonance imaging (fMRI) and positron emission tomography by numerous decades. These techniques are now becoming essential, both in physiological and pathological conditions, since they enable the imaging and quantification of organized active neural systems in working and resting states.

When an individual is awake and alert and not actively engaged in attention-

Received Nov. 8, 2011; revised Dec. 12, 2011; accepted Dec. 13, 2011.

We thank Daniela Perani, Gianvito Martino, Andrea Moro, Marco GalIoni, and William Cooke for their comments.

Correspondenceshould be addressed to Dr. Stefano Sandrone, Neuroimmunology Unit, Institute of Experimental Neurology, Division of Neuroscience, Via Olgettina 58, IRCCS San Raffaele Milano, I-20132 Milan, Italy. E-mail: sandrone.stefano@hsr.it.

D0I:10.1523/JNEUROSC1.5637-11.2012

Copyright $\odot 2012$ the authors $\quad 0270-6474 / 12 / 321915-03 \$ 15.00 / 0$ demanding tasks, organized neural activity is present in a set of brain regions called default mode network (DMN) (Raichle et al., 2001). This network involves densely interconnected pivotal structures, namely the posterior cingulate cortex (PCC), the precuneus, and parts of the ventromedial prefrontal cortex. DMN activity increases during lapses of attention, whereas it is suspended during specific goal-directed behaviors, a finding that implies that attention is sustained over time. In light of these characteristics, analysis of DMN activity can yield valuable insights about residual functional connectivity in neurological and psychiatric diseases.

In a paper recently published in The Journal of Neuroscience, Bonnelle et al. investigated the relationship between DMN and attentional tasks in traumatic brain injury (TBI) patients who suffer from diffuse axonal injury and difficulties in maintaining goal-directed behaviors because of impaired sustained attention (Bonnelle et al., 2011).

A group of post-acute/chronic traumatic patients, characterized by persistent cognitive problems, performed a choice reaction time (CRT) task during fMRI scanning. The subjects had to fixate on a cross on the screen (Rest trial) randomly interrupted by experimental blocks (Go trials) in which they were shown right or left triple arrows as "go" signals that signaled them to press a button with the right or left index finger as quickly and accurately as possible. The increase in reaction time (RT) over 144 trials was used to quantify the performance decrement of patients during the task. Although the experimental task may initially appear extremely simple, it becomes increasingly demanding as the trials progress, particularly because TBI patients suffer from variable sustained attention deficits.

The CRT task evoked DMN fluctuations between cognitive activity (decreased activity) and the resting state (increased activity). To investigate the impact of sustained attention deficits, Bonnelle et al. focused on cerebral activation differences across four conditions: Go and Rest trials during the first third (T1) of the session and Go and Rest trials during the last third (T3) of the session.

On the basis of the median of reaction time decrement of the individual patient over the course of the task, they identified two TBI subgroups: high- and lowsustained attention (SA). These groups did not differ in age, gender, education, presence of microbleeds, and time from injury. Injury severity was also comparable. The authors performed a standard voxelwise General Linear Model and a dual-regression functional connectivity analysis, a method that pursues not only spatial correlations but also time courses among brain areas to relate activation patterns to the DMN and another previously described brain system, the Executive Control/Salience network (Seeley et al., 2007). Compared to the high-SA group, low-SA showed a steeper increase in acti- 


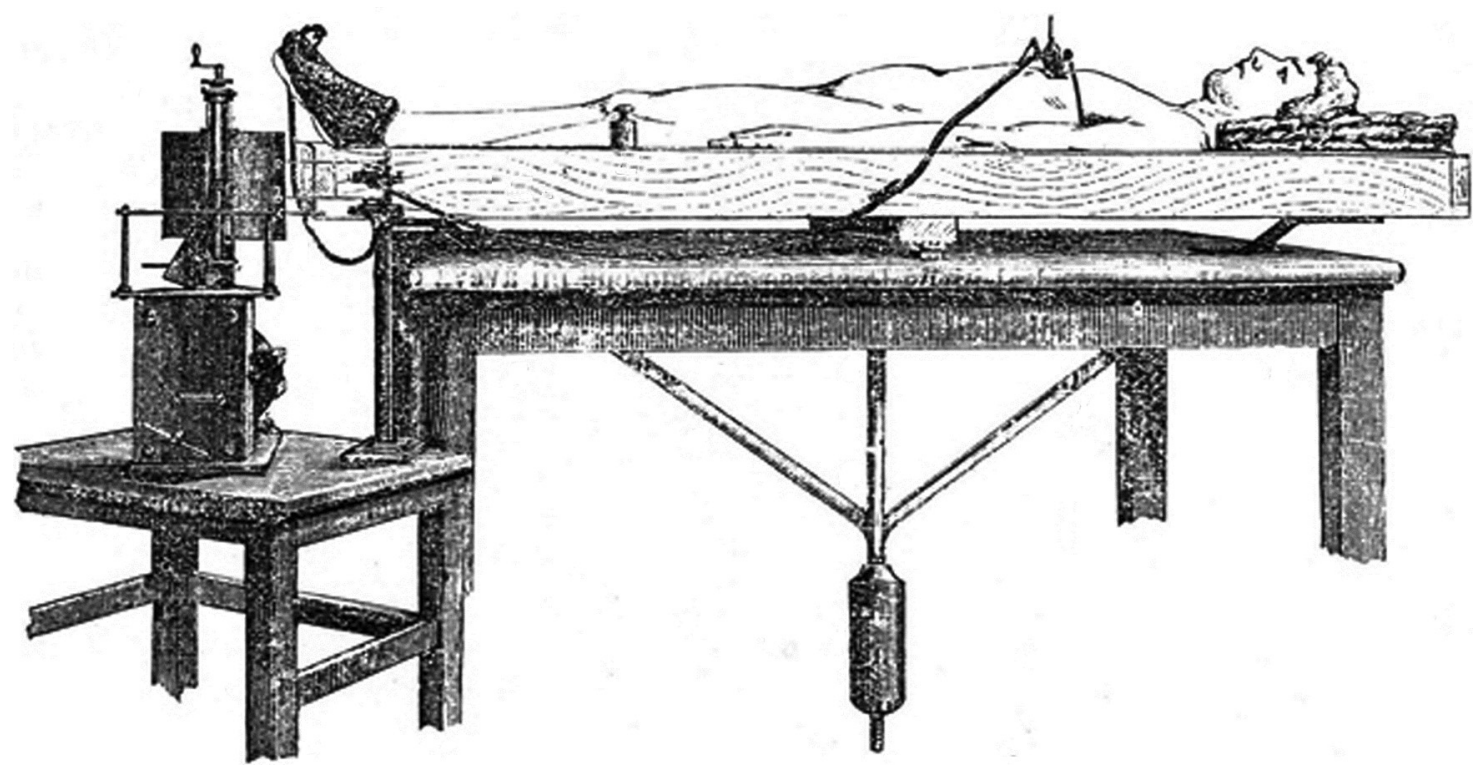

Figure 1. Human circulation balance used to measure cerebral activity during resting and cognitive states (Angelo Mosso's original drawing, modified and adapted from Mosso, 1884, Atti della Reale Accademia dei Lincei).

vation within the PCC from T1 to T3 in both the Go and the Rest trials. Furthermore, low-SA patients showed increased activation that also involved the precuneus in Rest trials and encompassed the left inferior parietal lobe as well as the left rostral superior frontal gyrus in Go trials. Altogether, these data confirmed that sustained attention impairments in patients are associated with an increase in DMN activation.

Perhaps the most interesting result obtained by Bonnelle et al. is that functional connectivity within the DMN can predict sustained attention ability in TBI patients. First, the authors correlated brain activation during T1 with the RT change over time and found that precuneus functional connectivity at T1 was strongly correlated with the change in RT over time in patients, but not in controls. Second, they used a stepwise logistic regression to determine whether measures of functional connectivity of the DMN structures at T1, i.e., before any behavioral evidence of attentional impairment, could predict the behavioral outcome. Keeping only the precuneus in the model allowed a correct classification of patients into high- and low-SA groups with $63 \%$ accuracy. Finally, using diffusion tensor imaging, Bonnelle et al. showed that structural disconnection within the DMN (in particular those of the right cingulum bundle) correlated with the level of sustained attention, which suggests that changes in connectivity within the DMN are crucial to the development of attentional impairment after TBI.
These results do not address theoretical controversies since they are compatible with the main hypotheses of the two opposite cognitive theories of vigilance decrement, as extensively discussed by the authors. Nonetheless, the performance prediction of high- and low-SA as based on a resting state measurement obtained before the emergence of any behavioral difference is striking. Certain caveats should nonetheless be highlighted. A prediction accuracy of $63 \%$ means that the proposed classification method is only $13 \%$ above the chance level. This is a limited predictive rate, and the limit could be related to the fact that there are some variables that have not been taken into account or that TBI is a highly heterogeneous pathology, or perhaps a combination of both.

In contrast with previous methodology (Sharp et al., 2011), the current study did not undertake any psychological assessments to measure the level of depression in the experimental subjects. This would have been interesting information, particularly because mood disturbances are common after TBI (Jorge and Robinson, 2003), and depression per se can affect both sustained attention performance (Godard et al., 2011) and DMN activation (Sheline et al., 2010).

Regarding heterogeneity, the study considered all the patients as having been sampled from a homogenous population despite various injury types and dynamics that included falls, assaults, traffic accidents, and sports-related events. However, this is not a sampling error. On the one hand, the execution of separate regression analyses considering subpopulations on the basis of injury type could theoretically have increased the prediction accuracy of the functional connectivity within the DMN by closer matching anatomical and thus functional variability between subjects. On the other hand, this stratification would have emptied the prediction on TBI patients of all logical and epistemological meaning, thus reducing a putative prediction to a mere description of the DMN. The beauty and interest of the prediction found by Bonnelle et al. is the fact that it goes over and beyond the heterogeneity of injury types, allowing the generalization to all TBI patients. DMN can be considered a promising marker for attentional performance, but the only way to validate it as a classification marker of attentional outcomes is to replicate this experiment on a larger sample.

From a more expansive perspective, we may say that this experiment explores one of the frontiers of current neuroscientific research in that it uses DMN abnormalities as a pathological marker and a clinical diagnostic tool with the aim of realizing a "functional taxonomy" of disease. Of pertinence here is the recent evidence that (1) DMN possibly plays a crucial role in early discrimination between similar dementias, such as between Alzheimer disease and dementia with Lewy bodies (Galvin et al., 2011), and (2) DMN may constitute a robust diagnostic or prognostic metric in individual patients with autism (Anderson et al., 2011). 
Observing the resting brain to predict a behavioral (and possibly clinical) outcome is a great challenge for neuroscience. Studies such as the one by Bonnelle and colleagues point in the right direction, but research on this issue is still in its infancy. Patience will be critical, and researchers will do well to learn Angelo Mosso's lesson: sometimes even a static person, whether lying on a "balanced table" or inside an fMRI scanner, can jolt neuroscientific research.

\section{References}

Anderson JS, Nielsen JA, Froehlich AL, Dubray MB, Druzgal TJ, Cariello AN, Cooperrider JR, Zielinski BA, Ravichandran C, Fletcher PT, Alexander AL, Bigler ED, Lange N, Lainhart JE (2011) Functional connectivity magnetic resonance imaging classification of autism. Brain 134:3739-3751.

Bonnelle V, Leech R, Kinnunen KM, Ham TE,
Beckmann CF, De Boissezon X, Greenwood RJ, Sharp DJ (2011) Default mode network connectivity predicts sustained attention deficits after traumatic brain injury. J Neurosci 31:13442-13451.

Galvin JE, Price JL, Yan Z, Morris JC, Sheline YI (2011) Resting bold fMRI differentiates dementia with Lewy bodies vs Alzheimer disease. Neurology 76:1797-1803.

Godard J, Grondin S, Baruch P, Lafleur MF (2011) Psychosocial and neurocognitive profiles in depressed patients with major depressive disorder and bipolar disorder. Psychiatry Res 190:244-252.

James W (1890) The Principles of Psychology. New York: Henry Holt.

Jorge R, Robinson RG (2003) Mood disorders following traumatic brain injury. Int Rev Psychiatry 15:317-327.

Mosso A (1884) Applicazione della bilancia allo studio della circolazione sanguigna dell'uomo (Application of the balance to the study of human brain circulation). Atti della Reale Acca- demia dei Lincei - Memorie della Classe di Scienze Fisiche, Matematiche e Naturali XIX:534.

Raichle ME, MacLeod AM, Snyder AZ, Powers WJ, Gusnard DA, Shulman GL (2001) A default mode of brain function. Proc Natl Acad Sci U S A 98:676-682.

Seeley WW, Menon V, Schatzberg AF, Keller J, Glover GH, Kenna H, Reiss AL, Greicius MD (2007) Dissociable intrinsic connectivity networks for salience processing and executive control. J Neurosci 27:2349-2356.

Sharp DJ, Beckmann CF, Greenwood R, Kinnunen KM, Bonnelle V, De Boissezon X, Powell JH, Counsell SJ, Patel MC, Leech R (2011) Default mode network functional and structural connectivity after traumatic brain injury. Brain 134:2233-2247.

Sheline YI, Price JL, Yan Z, Mintun MA (2010) Resting-state functional MRI in depression unmasks increased connectivity between networks via the dorsal nexus. Proc Natl Acad Sci U S A 107:11020-11025. 